\title{
Global land transport system ComplexTrans and its contribution to city sustainability
}

\author{
J. Hofman \\ ComplexTrans system Project, \\ University of West Bohemia, Czech Republic
}

\begin{abstract}
Global door-to-door land transport system ComplexTrans is based on mutual adaptation of road and rail vehicles.

This transport concept offers a breakthrough solution for the effective transport of passengers and cargo across medium and long distances. In addition, it improves the road network throughput and, up to the travel distance of 1000 $1500 \mathrm{~km}$, makes a viable substitute for continental air transport.

However, the required compatibility between rail and road vehicles entails profound changes to be made to the dimensions and character of both passenger and cargo road vehicles. These changes, on the other hand, result in new features and opportunities which promote sustainable development of cities through the following outcomes:

- reduced need for parking space for passenger cars;

- lower density of urban traffic;

- utilization of adapted passenger cars as comfortable and high-capacity boost to public transit;

- rapid elimination of exhaust gases and

- stabilization of power supply networks that use renewable energy sources. Keywords: road-rail, intermodal transport, improvement of city individual transport, public transport improvement, new solutions for parking, electrification of road transport, energy safety, support of renewable energy sources.
\end{abstract}

\section{Introduction}

Sustainable development of cities of the future is related to a number of aspects. These include the provision of congestion-free and safe individual and public 
transport for people between their homes and intended destinations, available parking spaces for passenger cars between trips, distribution of goods, clean air and stable power supply systems.

Those are also some of the goals of ComplexTrans - the global city and intercity door-to-door transport system for passengers and goods based on increased compatibility between road and rail vehicles.

At the heart of the ComplexTrans concept are new types of rail and road vehicles which cooperate instead of competing, and which combine the strengths of both modes of transport [1].

The advantages of the rail transport include greater safety, comfort and the maximum attainable speed. In addition, there is the use of electrical propulsion, lower running resistance, and its relative independence of weather conditions. Its drawbacks relate to the impossibility of door-to-door travel, less privacy, and schedule restrictions.

The strengths of the road transport consist in the availability of door-to-door travel, no time restrictions, and exclusively private space, whereas its weaknesses concern lower safety, frequent congestions, limited personal space during transport, and still prevalent dependence on fossil fuels.

Combination of both rail and road in one ComplexTrans system offers a transportation solution, which can provide predominantly the best travel conditions of all existing or known advanced transportation systems (fig. 1).

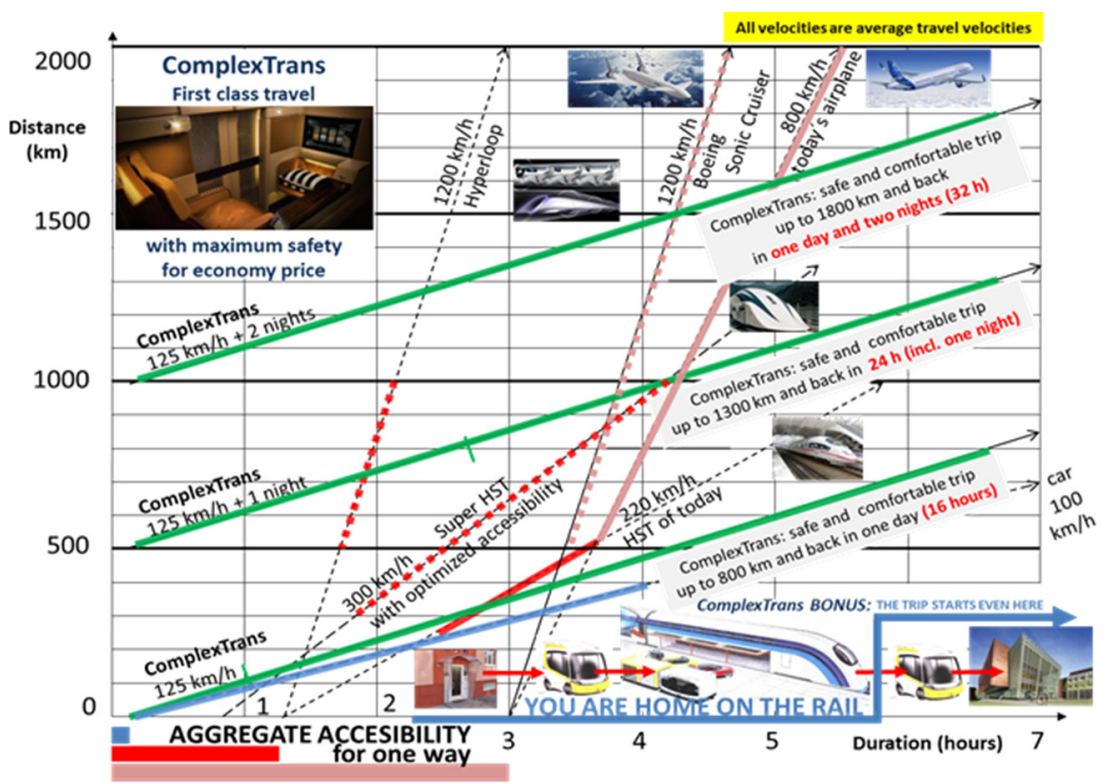

Figure 1: Comparison of ComplexTrans with other transportation systems.

The first pillar of the ComplexTrans transport concept is the integration of road and rail transport [1-13]. The initial and the final segments of travel are completed 
by road, whereas major part of the intercity distance is travelled in the adapted car transported by railway: on the lower deck of double-deck rail cars which the road vehicles can enter transversal during short stops of these trains at terminals [1-6, $9,12,13]$. Road and rail transport combined in this fashion provide rapid, comfortable and safe travel from door to door. In the rail car, the upper deck is designed for passenger transport $[1-6,12,13]$, whereas the lower deck serves freight transport, where the cargo is understood to consist of adapted passenger cars referred to as "coupemobiles", and of mobile transport modules for carrying goods or optionally for passengers (figs 2 and 3).

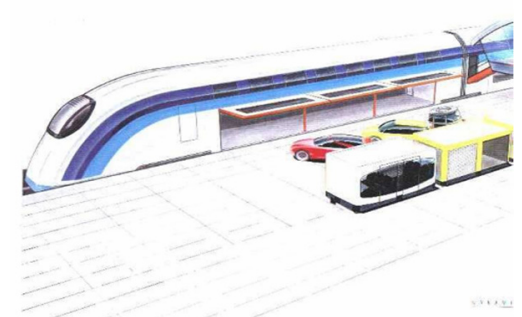

Figure 2: ComplexTrans train.

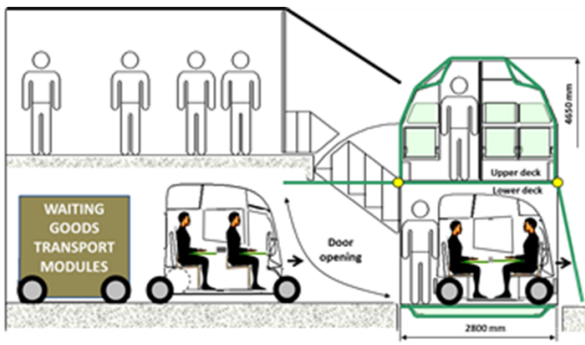

Figure 3: Loading of CT doubledeck coach.

The second pillar of the ComplexTrans concept is the simultaneous transport of passengers and mobile transport modules with cargo on the same train [1-6]. The transport modules with goods wait at the terminal for vacant slots on the train which have not been occupied by the passenger vehicles. This guarantees almost full utilization of the train's lower deck which is decisive for the economy of the operation of ComplexTrans trains (fig. 4). The joint transport of passengers and cargo eliminates freight trains which otherwise disrupt the service because they share the line with passenger trains. It also increases the frequency of trains thanks to larger volumes of transport. Higher train frequency reduces transport costs and minimizes waiting times, and therefore shortens travel times.

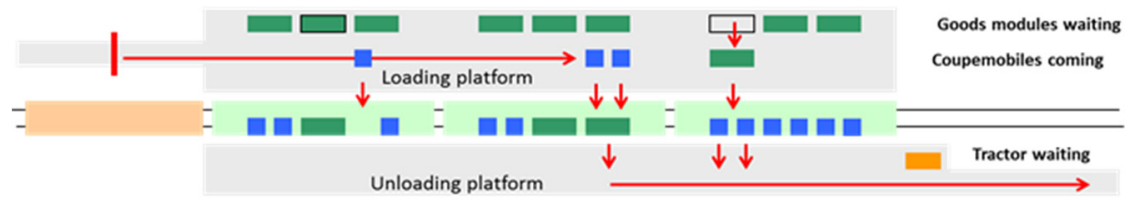

Figure 4: Disloading and loading of low deck of ComplexTrans train.

The third pillar of the ComplexTrans concept is the new form of a passenger car termed "coupemobile" [1-6] which shows similarities to a rail coach compartment (fig. 5) [14, 15]. Its design, alongside other technical features, permits sideways loading to, unloading from, and transport via ComplexTrans trains (fig. 3). When compared to conventional cars, coupemobiles are shorter, wider and higher vehicles with axles which can slide out forwards and rearwards 
[16]. Their drive unit is located under rear seats and their luggage compartment above them. They feature a different door configuration and a comfortable variable-layout interior which enables the usage for work, study, leisure or relaxation. Finally, coupemobiles are also adapted for vertical transport using manipulators and elevators (figs 7(b) and 7(c)). Coupemobiles are designed to be coupled and ride in sets which resemble buses (fig. 11).
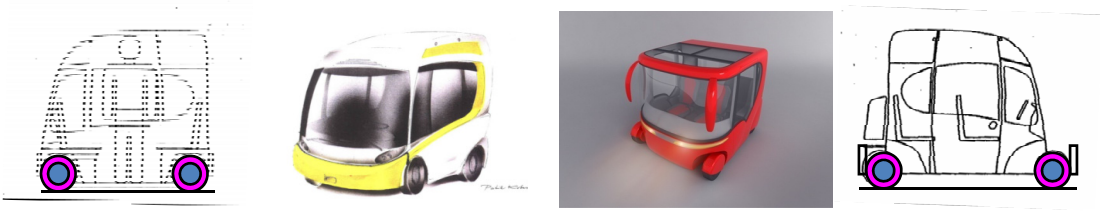

Figure 5: Coupemobile, the personal road vehicle of system ComplexTrans.

The fourth pillar of the ComplexTrans concept is mobile freight road modules which can be loaded onto ComplexTrans trains also from the side. They can also ride separately at limited speeds across limited distances in the terminal area or in sets guided by a lead vehicle across longer distances on roads. Mobile freight road modules are complemented by rapid freight rail cars which are transported either separately or in small sets at the end of a ComplexTrans train (fig. 6) [1-6].
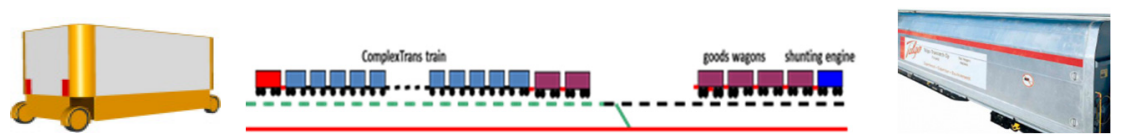

Figure 6: Goods transport module + fast goods wagons $=$ transport of goods in ComplexTrans trains.

All the above features and parameters that result from the alignment of road and rail transport open entirely not only new opportunities for long-distance transport, but they offer also valuable contribution to sustainable development of future cities.

\section{Seven contributions of ComplexTrans global transport concept to sustainable development of cities}

\subsection{Parking and stationary use of vehicles}

The parking of cars within cities poses several problems. Lines of cars spoil the appearance of city streets and reduce space for other activities. Lack of parking spaces and drivers' effort to find one increases the traffic density, disrupts the traffic flow and increases both energy consumption and greenhouse gas emissions. Car sharing can be thought of as a partial solution since better utilization of shared cars means reduced total numbers of cars. On the other hand, this solution is not the ultimate one because it does not address the issue of parking of private cars.

The ultimate solution, however, is offered by the ComplexTrans concept [16]. Given the great assets of the ComplexTrans concept, ordinary cars will be 
eventually replaced by coupemobile-type vehicles whose footprint of $2.2 \times 2 \mathrm{~m}$ (with their axles retracted) equals only about $60 \%$ that of an ordinary car. As a result, the need for parking areas will drop by approximately $40 \%$, not only for the shared but also for the private vehicles. The short length of the vehicle with the wheels retracted makes perpendicular parking possible (fig. 7(a)), and therefore shortens the parking manoeuvre and improves the parking area utilization [17]. Both outcomes deliver important contributions to solving parking problems and improving the traffic flow.

Coupemobiles boast yet another feature which makes parking easier. The vehicles are provided with standardized lugs and designed for being lifted by parking manipulators. In well-suited locations, vertical parking overground or underground towers or parking platforms above the pavement operated by manipulators can be built (fig. 7(b)). The increase in parking area obtained by this means is estimated at $20 \%$ at least.

Vertical transport of coupemobiles will be useful in multi-storey residential buildings. Elevators relocated outside the buildings will move coupemobiles by handling shoulder to and from parking spots on balconies where the vehicles will be safe from vandalism or theft and ready for effortless loading and unloading of luggage (fig. 7(c)).

By this means, the need for parking space will be substantially reduced.

Cars typically offer comfortable seats, heating, air-conditioning, lighting, highquality stereo audio systems and other features which are only used during travel. Parking on balconies will enable coupemobiles to be utilized as well-equipped small study rooms with a folding table, bedrooms, or for relaxation or listening to music.

The use of coupemobiles as work spaces will allow temporary office buildings to be established. Their ability to serve relaxation purposes will become the basis of simple and affordable accommodation services where the coupemobile will be integrated into the building.

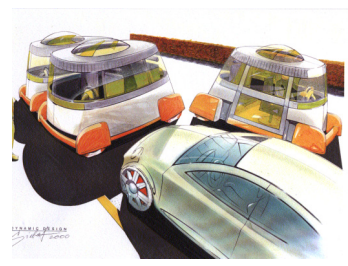

(a)

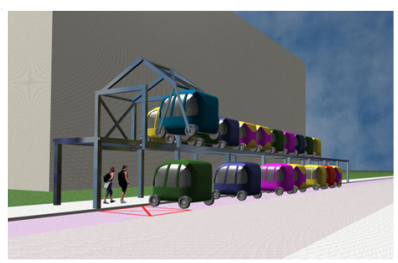

(b)

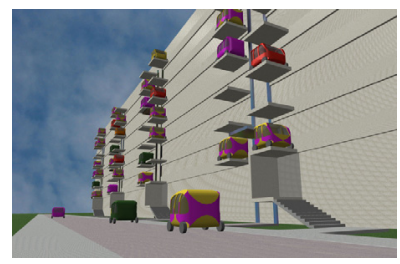

(c)

Figure 7: (a) Plumb parking; (b) Parking over pavements; (c) Balcony parking.

\subsection{Riding in sets}

Coupemobiles will be able to ride in tight sets. For some parts of the trip they will couple either mechanically or electronically with other coupemobiles travelling in the same direction. Riding in sets delivers multiple benefits $[1-6,18]$. First, the vehicles in the set do not need safe following distances between them, thus 
reducing traffic density. The required safe following distance between independent vehicles riding through a city is at least 15-20 metres. As a result, five moving vehicles take up 100 metres of the road lane at least. By contrast, five coupemobiles, each 3.2 metres long, coupled in a set, only take up approximately 40 metres, which includes the clearance ahead of the first coupemobile. By this means, traffic density can be reduced by up to two thirds.

In addition, vehicle sets improve intersection throughput because all vehicles start simultaneously and do not need to maintain long following distances. Similar to the previous case, the throughput of intersections can be doubled at least (fig. 8).
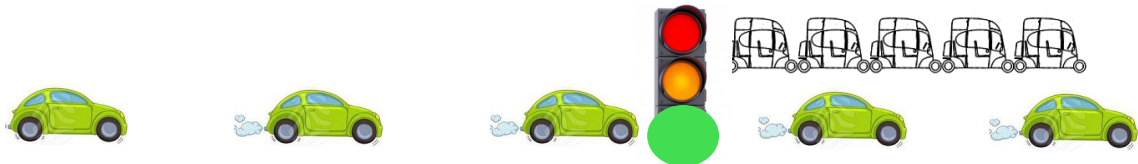

Figure 8: "Road trains" need less time for passing junctions.

An important advantage of riding in sets is the reduced energy consumption given by the fact that all vehicles in the set, except for the first one, face lower aerodynamic drag. At low speeds in urban environment, the aerodynamic drag is not severe but the saving can still be estimated at 10-20\%.

Riding in sets can deliver further benefits in the case of automatic control of road vehicles. Since only the lead vehicle needs to be controlled, whereas the others simply follow, the demand for data flows will be substantially reduced.

Coupemobiles will be able to couple electronically also during travel (fig. 10). Those prepared for coupling into sets will share information about their destination. Vehicles travelling to the same destinations will queue up and surrender control to the lead vehicle. To uncouple the vehicles, the set should preferably stop to allow all vehicles to assume independent control safely.

Another option for coupling the vehicles to sets will be mechanical coupling which is safer and provides even closer connection. Mechanical coupling to other vehicles will require stopping in a coupling lane, one possibility in fig. 9.

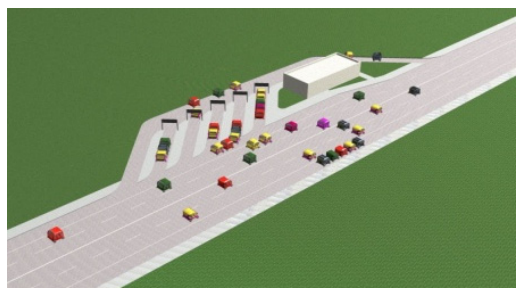

Figure 9: Place for mechanical coupling.

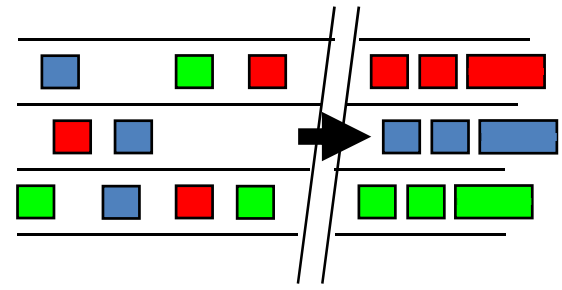

Figure 10:

Electronical coupling during ride.

\subsection{Vehicle sets in public transport}

Typically, a vehicle in the city (even in a set) only carries its driver. If the set consists of, say, five coupemobiles (fig. 11), each of which offers four comfortable 


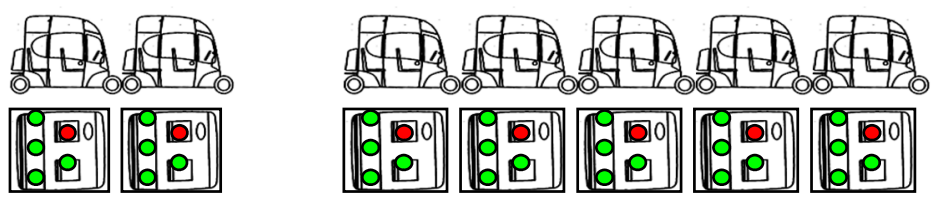

Figure 11: Sets of coupemobiles.

vacant seats, it becomes - in a sense - a bus with a capacity of twenty sitting passengers $[4,19]$.

Some of the drivers who use their passenger car for urban trips on a daily basis (e.g. to and from work) can be expected (20\%?) to be willing to offer unused seats in their coupemobiles as paid service for passengers travelling in the same direction $[4,20]$. In such case, they will enter into a contract with the transport authority which will provide the necessary equipment (namely travel pass readers and communication and navigation systems). While driving through the city, the coupemobile owner will report their preparedness to take part in public transport and their position and destination. The transport authority will identify travel requests from passengers via smartphones and match them with the capacity offered by coupemobile owners. Where the times and locations match, the navigation system will guide the necessary number of coupemobiles within tens of seconds to the first stop. There, the coupemobiles couple into a set, and the first passengers will board. The navigation system will then guide the set along a route which only includes the stops where passengers either board or alight (fig. 12). The transport organizer will ensure that the initially intended trip of each coupemobile does not extend by more than approx. $10 \%$. If the passenger demand increases steeply, the transport authority will invite contract coupemobile owners to start their travel earlier and better rewarded.

On their smartphone, each passenger will receive a message that lists in advance the time of arrival of their set, the reserved seat identification (the vehicle's location in the set and the seat), the approximate time of arrival in the destination and, if relevant, identification of transfer. When boarding and alighting, the passenger will touch the travel document with a chip on the validator, and confirm the scheduled data. This data will be used for calculating the compensation for coupemobile owners, and for operating the vehicle door. Another reason for employing chip cards is to ensure safe transport, where boarding will only be permitted for registered and screened persons.

This scheme of public transport offers a very comfortable service where the "bus" arrives on request to the most convenient stop, provides a reserved seat, stops only for boarding or alighting, benefits from traffic preferential features, and minimizes the time of travel and the number of transfers.

This public transport scheme will become particularly useful during peak hours when traffic preferential features will facilitate rapid travel of the coupemobiles to their destination, though slowed down by passenger boarding and alighting.

As a result, the size of the fleet operated by the transport authority will only need to cover the demand during traffic off-peak periods because the increased demand during peak hours will be met by coupemobiles (fig. 13). 
Integrating coupemobiles into public transit will have one more significant benefit: improved utilization of their capacity, and the associated reduction in urban traffic density. Finally, this will lead to lower energy demand of urban transport.

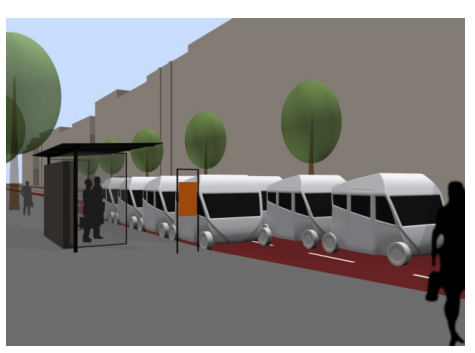

Figure 12: "Road train" in the station.

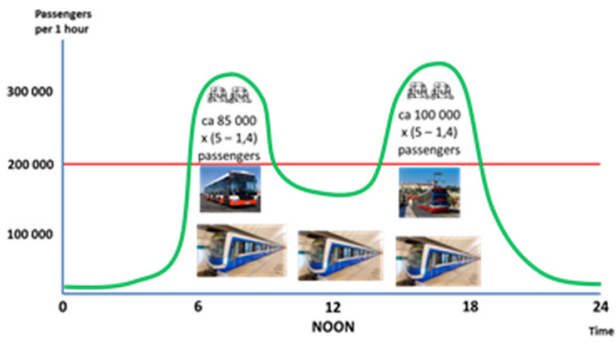

Figure 13: "Road trains" cover the peak time demand.

\subsection{Lightweight bridges}

To provide for effective combined operation of ComplexTrans trains and coupemobiles and also the cooperation between coupemobiles, a standard will be developed to govern the key dimensions and weights of vehicles, among other aspects. The key dimensions and parameters of coupemobiles will thus be identical. Therefore, it will be possible to construct tailored two-track lightweight bridges of optimized load-bearing and guide elements to run above intersections (fig. 14). As lightweight slender structures, these bridges will not spoil the appearance of the place where they will be installed.
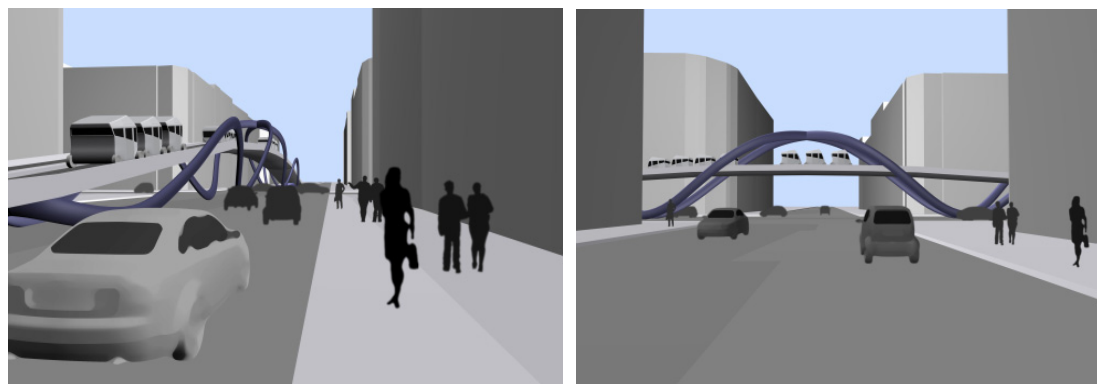

Figure 14: In unified dimensions, coupemobile's garnitures can use the light bridgings of junctions.

Access to them will be granted predominantly to those sets of coupemobiles that serve public transit purposes and, if applicable, to other coupemobile sets. The bridges will allow these sets to pass intersections without stopping, saving both time and energy. As a result, intersections will be easier to go also for other 
individual vehicles. The throughput of urban streets will be improved and congestion mitigated [4].

\subsection{Electric propulsion of cars}

We tend to expect the electric propulsion in cars to reduce their dependence on oil and the emissions of greenhouse and other harmful gases. Despite the recent progress in electric propulsion development, the low capacity of batteries (or large weight and volume) remain a major obstacle to the advancement of electric cars. Today's cars are required to provide the range of at least $500-600 \mathrm{~km}$ which equals 25-50 litres of gasoline (weighing 20-40 kg), depending on the car's weight and speed, the road slopes, the driving style and other parameters. To provide such range, however, a battery produced by current technologies would have to weigh ten to twenty times more, which would, in turn, increase the energy consumption and in fact, also increase the required weight and size of the battery. Other difficulties lie in the high price, limited life and long charging times by conventional methods. All these aspects delay the widespread use of electric propulsion. It is possible that finding an alternative energy storage suitable for cars will yet take a long time.

The ComplexTrans concept enables road vehicles to cross large distances by railway (without consuming energy from their own storage and even replenish their energy storage). Cars (coupemobiles) can therefore do with an energy storage capacity (approx. $30 \mathrm{kWh}$ ) for a range 100 to $150 \mathrm{~km}$ because the distance they travel in urban traffic in a single day will not be longer, and at night their storage will be replenished. This recharging can also be met easier by parking on balconies or in other parking installations where electrical outlets will commonly be available for recharging the vehicle's batteries (figs 7(b) and 7(c)). Consequently, the electric energy storage required for cars (coupemobiles) operated in the ComplexTrans system will be relatively small, in comparison to those needed for cars today. The ComplexTrans therefore enables electric propulsion in cars even today (fig. 15) [5].
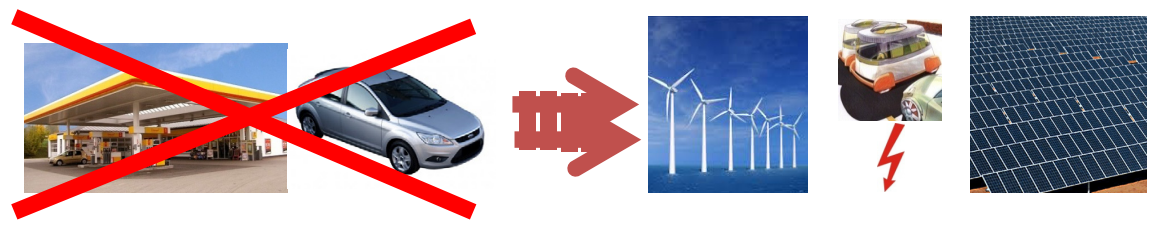

Figure 15: Thanks to ComplexTrans, trains can make the battery of coupemobiles smaller and the electrification of road transport faster.

For an independent long-distance ride of a coupemobile on a road, a supplementary energy source to be fitted to the back of the vehicle will be available for rent at petrol stations. It may take the form of a petrol generator [21].

\subsection{Integration with power supply network}

As mentioned in the preceding section, coupemobiles will connect to the power supply network when parked and even when transported by train, and therefore 
they will be able to recharge. This option offers not only service for the cars' electric propulsion but also an opportunity for the power supply network to make use of energy storage in the cars.

For each 10 million people, there are about 5 million passenger cars. If these cars are replaced with electric coupemobiles equipped with $30 \mathrm{kWh}$-capacity batteries, and these batteries are recharged at $6 \mathrm{~kW}$ for 5 hours, their total power input will be roughly equal to $60 \%$ of the capacity of the power supply network adapted for electric propulsion of cars (approx. 50,000 MW).

As one third of coupemobiles connect to the power supply network while parked or being carried on a train, their batteries can absorb $20 \%$ of the total capacity of all power plants (fig. 16). By this means, the problems of excess supply from volatile renewable sources, such as solar and wind farms, can be mitigated [5].

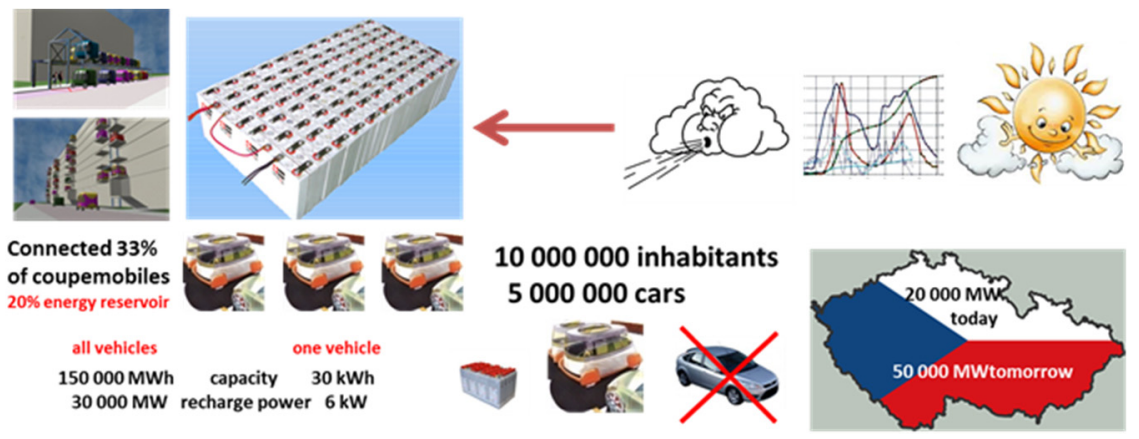

Figure 16: Batteries of $1 / 3$ coupemobiles represent a $20 \%$ energy storage of all energy resources.

Conversely, when the supply cannot meet the demand, coupemobile batteries can be discharged for a compensation fee in order to provide energy for the power supply network. For the coupemobile owner, this compensation can provide some revenue for their contribution to network stability at the time of lack of supply or power generation outage.

Introduction of electric propulsion in cars facilitated by the launch of the ComplexTrans system could provide a mutually beneficial close connection between land transport and power supply network.

\subsection{Transport of goods}

Shops and companies in urban areas are supplied using trucks and vans which have to arrive at the point of unloading or loading. This mostly takes place during their drivers' working hours during the day, often at unfavourable times when these activities constrain other traffic.

Under the ComplexTrans concept [1-6], road transport of goods is provided by unmanned transport modules (fig. 18), which, having been unloaded from the train, wait on the parking lot at the terminal for the right time to make the trip to their destination (fig. 17). In the off-peak hours, the transport modules travel as 
short sets guided by the lead vehicle (fig. 19), with a driver to their destinations and are handed over to customers or even parked in a suitable location without any contact with the customer. Final handling operations across tens or hundreds of metres and unloading or loading are completed by the customer using the drive which is built in the goods module.

The loaded or empty transport module then waits for its time to be transported to the terminal or to another customer in a set guided by a lead vehicle with a driver.

Understandably, urgent deliveries can be completed during peak hours. In such case, the modules might travel as single and not in sets.

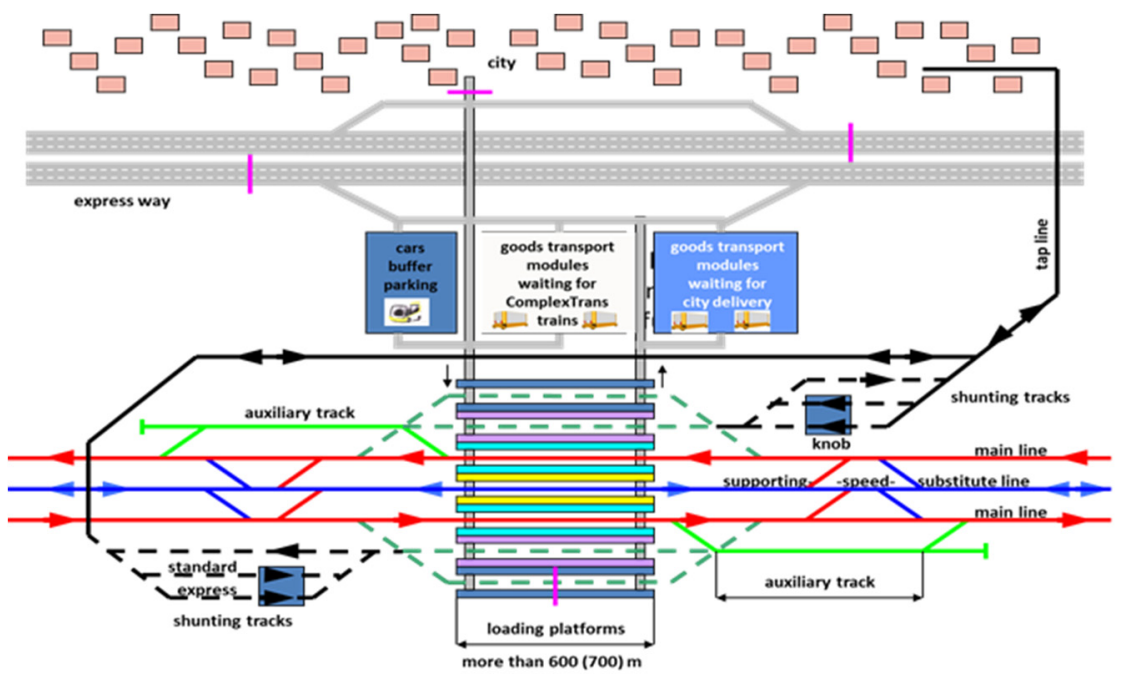

Figure 17: ComplexTrans terminal with parking areas for waiting goods transportation modules.

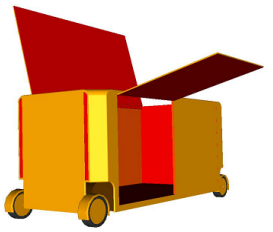

Figure 18: Goods transport module.

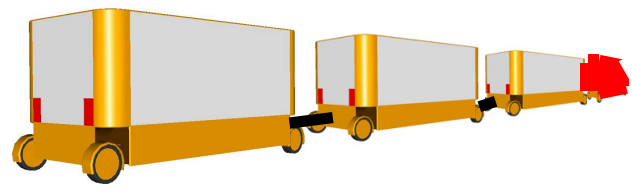

Figure 19: Goods transport modules going to /from the customer.

\section{Conclusion}

Although the initial motivation for the ComplexTrans global transport concept was to provide comfortable, rapid and safe transport of passengers and cargo from door to door across large distances, independently of weather conditions and fossil 
fuels, the necessary alterations to dimensions, properties and character of both passenger and cargo vehicles will enable ComplexTrans to introduce numerous changes to cities, contributing to their sustainable development.

These change include, above all, less need for parking areas, non-transport utilization of vehicles, reduced urban traffic intensity without reducing the transport performance, a new effective and comfortable supplementary system of public transit based on efficiently organized trips of private vehicles, and finally an extensive use of electric propulsion in vehicles leading to improved air quality in cities, and support for renewable energy sources and higher energy security.

For sustainable development of cities brings the global transportation system ComplexTrans quite new interesting possibilities.

\section{References}

[1] Hofman J., Koenig V., WO9929552, https://worldwide.espacenet.com/ publicationDetails/biblio? $\mathrm{DB}=\mathrm{EPODOC} \& \mathrm{II}=16 \& \mathrm{ND}=3 \&$ adjacent $=$ true $\& 1$ ocale $=$ en $E P \& F T=D \&$ date $=19990617 \& C C=W O \& N R=9929552 A 2 \& K C=$ A2 1999

[2] CKD, Bertone Design, Hannover University, Skoda Transportation, VUKV - application to FP5 (EC) Growth - GRD1-2000-25476, 2000

[3] Hofman J., Korecky M. - 37. Moderne Schienenfahrzeuge, http://www.schienenfahrzeugtagung.at/37 tagung.htm Graz, 2007

[4] Hofman J., UITP GoSmart-GoRail, Munich, 2015

[5] Hofman J., Transport Day, COP21 clima conference, Paris, 2015

[6] Hofman J., Linkedin - https://www.linkedin.com/in/hofmanjiri list of conferences and publications

[7] Container Train https://en.wikipedia.org/wiki/Rail freight transport \#Containerization

[8] Rolling Motorway http://www.sbbcargo-international.com/en/rollinghighway-rola. html

[9] Lohr, Transport of semi-trailers, http://lohr.fr/lohr-railway-system-en/thelohr-system-terminals/ 2014

[10] Car train https://www.sbb.ch/en/station-services/car-bike/car-trains/ autoreisezug.html

[11] Eurotunnel, https://www.eurotunnel.com/uk/home/ 1994

[12] Riessberger K., Prof., Technical report 85-53, 85-62 TU Graz Eisenbahnwesen, 1985

[13] Rinderknecht F.M., Rinspeed UC Konzept, http://www.auto.at/contator/ auto/news.asp?nnr=41489 2003

[14] Honda, Puyo, http://www.autoblog.com/2007/10/09/tokyo-2007-previewhonda-puyo-concept-wants-you-to-touch-it/ 2007

[15] Toyota, Rin, http://www.toyota-global.com/showroom/toyota design/ concept cars/gallery toyota rin.html 2007

[16] Matra-Renault, Zoom - with retractable back axle, https://en.wikipedia.org /wiki/Renault Zoom 1992

[17] Hofman J., Minimobil, http://evworld.com/news.cfm?newsid=8899 2005 
[18] Toyota, PM - connected cars, http://www.gizmag.com/go/2314/pictures 2003

[19] Lohr, Cristal Strassbourg, http://www.climatesolutionsplatform.org/solution /cristal-new-dual-mode-transport-system-electric-economic-digital/ 2015

[20] Uber https://www.uber.com/

[21] EP Tender http://www.eptender.com/ 